\title{
COMPACT MEASURES HAVE LOEB PREIMAGES
}

\author{
DAVID ROSS
}

(Communicated by Andreas R. Blass)

\begin{abstract}
A compact measure is a (possibly nontopological) measure that is inner-regular with respect to a compact family of measurable sets. The main result of this paper is that every compact probability measure is the image, under a measure-preserving transformation, of a Loeb probability space. This generalizes a well-known result about Radon topological probability measures. It is also proved that a compact probability space can be topologized in such a way that the measure is essentially Radon.
\end{abstract}

\section{INTRODUCTION}

Which probability measures can be represented by a Loeb space?

In [2] Robert Anderson proved that every Radon probability measure on a Hausdorff space $X$ is the image, under the standard part map, of a Loeb measure on ${ }^{*} X$. Depending on the topology on $X$, a converse can be proved; for example, D. Landers and L. Rogge [4] prove: if $X$ is regular, then every probability measure, which is the image under the standard part map of a Loeb measure on ${ }^{*} X$, is Radon.

The situation when $X$ is not a Hausdorff topological space is more problematic, as the standard part map is not defined. An early approach to representing nontopological probability spaces by the Loeb measure [5] dispenses with measurable transformations altogether, but does not appear to have much application. The case when $X$ is topological but not Hausdorff would seem to be easier than the general case, but still suffers from the lack of a standard part map (although T. Norberg [7] has recently constructed a natural analogue of the standard part map for so-called 'sober' spaces). Such spaces arise naturally in extremal theory and the theory of random closed sets (see e.g., [8] or [11]).

In this paper I consider a nontopological analogue of a Radon space, called a compact probability space. The main theorem is that every compact probability space is the image, under a measure-preserving transformation $\varphi$, of a Loeb space. $\varphi$ is very easy to construct, and acts very much like the standard part map; in particular, it is used to define a topology on $X$ with respect to which $\varphi$ is the standard part map, provided this topology is Hausdorff.

Received by the editors August 17, 1990 and, in revised form, December 3, 1990.

1980 Mathematics Subject Classification (1985 Revision). Primary 28E05; Secondary 28C99, $03 \mathrm{H} 05$.

Key words and phrases. Loeb measure, compact measure, Radon measure. 
A secondary, and in statement entirely standard, result is that every compact probability space can be topologized so as to be compact-inner-regular; by extending the $\sigma$-algebra and the domain of the measure a bit, one can make the probability space Radon.

\section{Statement of Results}

Let $X$ be any set, and $\mathscr{A} \subseteq \mathscr{P}(X)$.

$\mathscr{A}$ is centered if $\cap \mathscr{A} \neq \varnothing$.

$\mathscr{A}$ has the finite intersection property if every finite subset of $\mathscr{A}$ is centered.

$\mathscr{A}$ is $\alpha$-compact ( $\alpha$ a cardinal) provided whenever $\mathscr{A}^{\prime} \subseteq \mathscr{A}$ has the finite intersection property and cardinality $\leq \alpha, \mathscr{A}^{\prime}$ is centered.

$\mathscr{A}$ is compact if it is $\alpha$-compact for every cardinal $\alpha$.

For example, the collection of compact subsets of a topological space is compact. Another example: if $X$ is an internal set in a $\kappa^{+}$-saturated nonstandard model, then ${ }^{*} \mathscr{P}(X)$ is $\kappa$-compact.

If $\mathscr{A}$ is $(\alpha-)$ compact then clearly so is $\mathscr{A} \cup\{X\}$; it is assumed for the remainder of this paper that $X \in \mathscr{A}$ whenever $\mathscr{A}$ is said to be compact.

A probability space $(X, \mathscr{B}, P)$ is $\alpha$-compact provided there is an $\alpha$-compact set $\mathscr{K} \subseteq \mathscr{B}$ with $P(E)=\sup \{P(K): K \in \mathscr{K}, K \subseteq E\}$ (in other words, if $P$ is $\mathscr{K}$-inner regular). The role of $\mathscr{K}$ is often emphasized by calling the probability space $\mathscr{K}-(\alpha-)$ compact.

Note that many authors use the term 'compact' as a synonym for $\aleph_{0}$-compact (e.g., [9]).

Given $(X, \mathscr{B}, P)$, denote by $\overline{\mathscr{B}}$ the completion of $\mathscr{B}$ with respect to $P$; note that if $(X, \mathscr{B}, P)$ is $\mathscr{K}$-compact, then so is $(X, \overline{\mathscr{B}}, P)$.

Suppose now that $X$ is a topological space with (not necessarily Hausdorff) topology $\tau$. Let $\mathscr{B}(\tau)$ be the smallest $\sigma$-algebra containing $\tau$, and let $\mathscr{K}(\tau)=$ $\{K \subseteq X: K$ is compact (relative to $\tau)\}$. A probability space $(X, \mathscr{B}(\tau), P$ ) is Radon provided it is $\mathscr{K}(\tau)$-compact. As this term is normally applied only to measures on Hausdorff topological spaces, the first assertion of Theorem 1.1 below is stronger than known results.

The reader is assumed to be familiar with the Loeb measure construction; see [1], [3], or [6]. Every Loeb probability space in this paper is complete, and the nonstandard model is saturated in the cardinality of every standard set that arises.

Call a probability space $\mathscr{K}$-supercompact if it is $\mathscr{K}$-compact where $\mathscr{K}$ is closed under finite unions and arbitrary intersections.

Theorem 1.1. Suppose $(X, \mathscr{B}, P)$ is a $\mathscr{K}$-compact probability space.

(i) There is a measure-preserving surjection

$$
\varphi:\left({ }^{*} X, L\left({ }^{*} \mathscr{B}\right), L\left({ }^{*} P\right)\right) \rightarrow(X, \mathscr{B}, P) .
$$

(ii) For some $\mathscr{B}^{\prime} \supseteq \mathscr{B}, \mathscr{K}^{\prime} \supseteq \mathscr{K}$, and extension $P^{\prime}$ of $P$ to $\mathscr{B}^{\prime},\left(X, \mathscr{B}^{\prime}, P^{\prime}\right)$ is a $\mathscr{K}^{\prime}$-supercompact probability space.

(iii) If $(X, \mathscr{B}, P)$ is $\mathscr{K}$-supercompact then for some topology $\tau$ on $X$, $\mathscr{B}(\tau) \subseteq \mathscr{B} \subseteq \overline{\mathscr{B}}(\tau)$ and $\mathscr{K} \subseteq \mathscr{K}(\tau)$; in particular, $(X, \mathscr{B}, P)$ is Radon.

The proof in $\S 4$ actually yields more; namely, when $(X, \mathscr{B}, P)$ is extended in (ii) to a $\mathscr{K}$-supercompact space, and the topology $\tau$ is found for (iii), then 
$\mathscr{B}$ is in the completion of $\mathscr{B}(\beta)$, where $\beta$ is a basis for $\tau$. In other words, the relation between the supercompact space $\left(X, \mathscr{B}^{\prime}, P^{\prime}\right)$ and the compact space $(X, \mathscr{B}, P)$ is something like the relation of a Radon measure to the Baire measure obtained by restriction to the Baire algebra.

\section{THE TOPOLOGY $\tau_{\varphi}$}

In this section $X$ is any set, $S \subseteq{ }^{*} X$ is arbitrary, and $\varphi: S \rightarrow X$ is a surjection. Call $E \subseteq X \quad \varphi$-open provided $\varphi^{-1}(E) \subseteq{ }^{*} E$, and $\varphi$-closed if $E^{c}$ is $\varphi$-open (equivalently, if $S \cap^{*} E \subseteq \varphi^{-1}(E)$ ). Put $\tau_{\varphi}=\{u \subseteq X: u$ is $\varphi$-open $\}$.

Lemma 2.1. (i) $\tau_{\varphi}$ is a topology on $X$.

(ii) If $E$ is $\varphi$-closed, $X \subseteq S$, and $S \cap{ }^{*} E$ is internal, then $E \in \mathscr{K}\left(\tau_{\varphi}\right)$.

Proof. (i) Clearly $\varnothing, X \in \tau_{\varphi}$. If $u, v \in \tau_{\varphi}$ then $\varphi^{-1}(u \cap v)=\varphi^{-1}(u) \cap$ $\varphi^{-1}(v) \subseteq{ }^{*} u \cap{ }^{*} v={ }^{*}(u \cap v)$, so $\tau_{\varphi}$ is closed under finite intersections. If $\left\{u_{i}\right\}_{i \in I} \subseteq \tau_{\varphi}$ then $\varphi^{-1}\left(\bigcup_{I} u_{i}\right)=\bigcup_{I} \varphi^{-1}\left(u_{i}\right) \subseteq \bigcup_{I}{ }^{*} u_{i} \subseteq{ }^{*}\left(\bigcup_{I} u_{i}\right)$, so $\tau_{\varphi}$ is closed under arbitrary unions; this makes $\tau_{\varphi}$ a topology on $X$.

(ii) Let $\left\{u_{i}\right\}_{i \in I} \subseteq \tau_{\varphi}$ with $E \subseteq \bigcup_{I} u_{i}$. Then $S \cap^{*} E \subseteq \varphi^{-1}(E) \subseteq \varphi^{-1}\left(\bigcup_{I} u_{i}\right)=$ $\bigcup_{I} \varphi^{-1}\left(u_{i}\right) \subseteq \bigcup_{I}{ }^{*} u_{i}$. Since $S \cap{ }^{*} E$ is internal, and the model is card $(I)$ saturated, $S \cap{ }^{*} E \subseteq{ }^{*} u_{i_{1}} \cup \cdots \cup \cup^{*} u_{i_{n}}$ for some finite $\left\{i_{1}, \ldots, i_{n}\right\} \subseteq I$. If $x \in E$ then $x \in S \cap{ }^{*} E \subseteq{ }^{*}\left(u_{i_{1}} \cup \cdots \cup u_{i_{n}}\right)$; by transfer, $x \in u_{i_{1}} \cup \cdots \cup u_{i_{n}}$. It follows that $E \subseteq u_{i_{1}} \cup \cdots \cup u_{i_{n}}$. Since $\left\{u_{i}\right\}_{i \in I}$ was an arbitrary open cover of $E$, $E \in \mathscr{K}\left(\tau_{\varphi}\right)$.

Note the following: (1) The hypotheses " $S \cap{ }^{*} E$ is internal" in this lemma can be replaced by " $S \cap{ }^{*} E$ is the intersection of $\kappa$ many internal sets" when the model is $\kappa^{+}$-saturated.

(2) If $S={ }^{*} X$ then every $\varphi$-closed set, including $X$, is in $\mathscr{K}\left(\tau_{\varphi}\right)$. This is the case for the remainder of the paper.

(3) When $\tau_{\varphi}$ is not Hausdorff, then the elements of $\mathscr{K}\left(\tau_{\varphi}\right)$ need not be closed.

(4) If $\tau_{\varphi}$ is Hausdorff, $X \subseteq S$, and $\varphi(x)=x$ for all $x \in X$, then $\varphi$ is the standard part map on $S$.

\section{COMPACT FAMILIES}

Suppose $X$ is any set, and that $\mathscr{K} \subseteq \mathscr{P}(X)$ is compact. Define a set function $\varphi_{\mathscr{K}}:{ }^{*} X \rightarrow X$ by $\varphi_{\mathscr{K}}(x)=\cap\left\{K \in \mathscr{K}: x \in{ }^{*} K\right\}$. Since $\mathscr{K}$ is compact, $\varphi_{\mathscr{K}}(x)$ is always nonempty. A function $\varphi:{ }^{*} X \rightarrow X$ with $\varphi(x) \in \varphi_{\mathscr{K}}(x)$ for all $x$ is a selection of $\varphi_{\mathscr{K}}$; if in addition $\varphi(x)=x$ for all $x \in X$ then $\varphi$ is a good selection. Note that every good selection is a surjection; moreover, since $x \in \varphi_{\mathscr{K}}(x)$ for every $x \in X$, good selections always exist.

Lemma 3.1. Let $\mathscr{K} \subseteq \mathscr{P}(X)$ be compact, and let $\varphi$ be a good selection of $\varphi_{\mathscr{K}}$. Let $K \in \mathscr{K}$. Then:

(i) $\varphi\left({ }^{*} K\right)=K$;

(ii) $K$ is $\varphi$-closed;

(iii) $K \in \mathscr{K}\left(\tau_{\varphi}\right)$.

Proof. (i) If $x \in{ }^{*} K$ then $\varphi(x) \in \varphi_{\mathscr{K}}(x) \subseteq K$ by definition of $\varphi_{\mathscr{K}}$. Conversely, if $x \in K$, then $x=\varphi(x) \subseteq \varphi(K) \subseteq \varphi\left({ }^{*} K\right)$, so $\subseteq$ holds.

(ii) Immediate from (i). 
(iii) By (ii) and Lemma 2.1.

Corollary 3.2. Suppose $\mathscr{K} \subseteq \mathscr{P}(X)$ is compact. Then:

(i) Every subset of $\mathscr{K}$ is compact.

(ii) The closure of $\mathscr{K}$ under finite unions is compact.

(iii) The closure of $\mathscr{K}$ under arbitrary intersections is compact.

Proof. (i) is clear; (ii) and (iii) follow since every element of $\mathscr{K}$ is $\varphi$-closed, the collection of $\varphi$-closed sets is closed under finite unions, arbitrary intersections, and by Lemma 3.1 is a subset of the compact collection $\mathscr{K}\left(\tau_{\varphi}\right)$.

Consider an example. Suppose $X=\mathbb{R}$, and $\mathscr{K}$ is the collection of compact subsets of $\mathbb{R}$. For $x$ a nearstandard element of ${ }^{*} \mathbb{R}$, local compactness of $\mathbb{R}$ ensures that $\varphi(x)=\operatorname{st}(x)$. If $x$ is not nearstandard then $\varphi(x)$ can be anything at all; for convenience, suppose $\varphi(x)=0$ for such $x$. Evidently $\tau_{\varphi}$ consists of those usual open subsets of $\mathbb{R}$ that do not contain 0 , together with all complements of compact sets. Note that $\tau_{\varphi}$ clearly depends on the particular choice of $\varphi$.

It is easy to see directly why $\mathbb{R}$ is compact in this topology. Any open cover $\mathscr{U}$ contains an element $u$ with $0 \in u$. Then $u^{c}$ is compact, and so has a finite subcover from $\mathscr{U}$. This subcover, together with $u$, covers $\mathbb{R}$.

\section{Proof of TheOREM 1.1}

By Corollary 3.2 it may be assumed that $\mathscr{K}$ is closed under finite unions. Let $\varphi$ be a good selection of $\varphi_{\mathscr{K}}$.

If $E \in \mathscr{B}$ and $\varepsilon>0$, then there are $K, K^{\prime} \in \mathscr{K}$ with $K \subseteq E, K^{\prime} \subseteq E^{c}$, and $P(E \backslash K)+P\left(E^{c} \backslash K^{\prime}\right)<\varepsilon$. Put $U=\left(K^{\prime}\right)^{c} \in \tau_{\varphi}$; then ${ }^{*} K \subseteq \varphi^{-1}(K) \subseteq$ $\varphi^{-1}(E) \subseteq \varphi^{-1}(U) \subseteq{ }^{*} U$ and ${ }^{*} P\left({ }^{*} U \backslash^{*} K\right)<\varepsilon$. Since $\varepsilon$ is arbitrary, and by assumption $L\left({ }^{*} P\right)$ is complete, $\varphi^{-1}(E)$ is $L\left({ }^{*} P\right)$ measurable with measure $P(E)$. This proves $(\mathrm{i})$.

Let $\beta=\left\{K^{c}: K \in \mathscr{K}\right\}$; since $\mathscr{K}$ is closed under finite unions, $\beta$ is closed under finite intersections, and so forms a basis for a topology $\tau$. By Lemma 3.1, $\mathscr{K} \subseteq \mathscr{K}\left(\tau_{\varphi}\right)$, and since $\tau \subseteq \tau_{\varphi}, \mathscr{K}\left(\tau_{\varphi}\right) \subseteq \mathscr{K}(\tau)$. A typical $\tau$-closed set has the form $E=\cap_{I} K_{i}$ where each $K_{i} \in \mathscr{K}$. If in fact $(X, \mathscr{B}, P)$ is $\mathscr{K}$-supercompact then $E \in \mathscr{K} ;$ since $\mathscr{K} \subseteq \mathscr{B}$, (iii) is immediate.

Suppose for (ii), that $(X, \mathscr{B}, P)$ is not $\mathscr{K}$-supercompact. Let $\mathscr{B}^{\prime}$ be the smallest $\sigma$-algebra containing $\mathscr{B}(\tau) \cup \mathscr{B}$, and let $\mathscr{K}^{\prime}$ be the closure of $\mathscr{K}$ under arbitrary intersections; in other words, $\mathscr{K}^{\prime}$ is the collection of $\tau$-closed subsets of $X$. By Corollary 3.2, $\mathscr{K}^{\prime}$ is compact. It remains to extend $P$ to a $\mathscr{K}^{\prime}$-inner regular probability measure defined on all of $\mathscr{B}^{\prime}$.

As above, let $E=\bigcap_{I} K_{i}$, where each $K_{i} \in \mathscr{K}$. Without loss of generality, $\left\{K_{i}\right\}_{i \in I}$ is closed under finite intersections. If $n \in I$, then $P\left(K_{n}\right)=$ $L\left({ }^{*} P\right) \varphi^{-1}\left(K_{n}\right) \geq \overline{L\left({ }^{*} P\right)} \varphi^{-1}\left(\bigcap_{I} K_{i}\right) \geq \underline{L\left({ }^{*} P\right)}\left(\bigcap_{I} \varphi^{-1}\left(K_{i}\right)\right) \geq \underline{L\left({ }^{*} P\right)}\left(\bigcap_{I}{ }^{*} K_{i}\right)$. (Here $\overline{L\left({ }^{*} P\right)}$ and $L\left({ }^{*} P\right)$ are the Loeb inner and outer measures on ${ }^{*} X$.) From a result of Landers and Rogge [4], $\bigcap_{I}{ }^{*} K_{i}$ is $L\left({ }^{*} P\right)$-measurable with $L\left({ }^{*} P\right)\left(\bigcap_{I}{ }^{*} K_{i}\right)=\inf _{i \in I} L\left({ }^{*} P\right)\left({ }^{*} K_{i}\right)=\inf _{i \in I} P\left(K_{i}\right)$. Since $n \in I$ is arbitrary, $\bigcap_{I} \varphi^{-1}\left(K_{i}\right)=\varphi^{-1}(E)$ is measurable with measure $\inf _{i \in I} P\left(K_{i}\right)$.

As $\varphi$ is therefore measurable with respect to $\mathscr{B}(\tau)$, it is measurable with respect to all of $\mathscr{B}^{\prime}$, and is measure-preserving if one puts $P^{\prime}(E)=L\left({ }^{*} P\right) \varphi^{-1}(E)$ for $E \in \mathscr{B}^{\prime}$. It remains to show that $P^{\prime}$ is $\mathscr{K}^{\prime}$-inner regular. 
Call $A \subseteq X$ approximable if for all $\varepsilon>0$ there exist $E \in \mathscr{K}^{\prime}$ and $u \in \tau$ such that $E \subseteq A \subseteq u$ and $P(u \backslash E)<\varepsilon$. Let $\mathscr{A}=\{A \subseteq X \mid A$ approximable $\} . P^{\prime}$ is $\mathscr{K}^{\prime}$-inner regular if $\mathscr{B}^{\prime} \subseteq \mathscr{A}$; as $\mathscr{B} \subseteq \mathscr{A}$ it suffices to show that $\mathscr{K}^{\prime} \subseteq \mathscr{A}$, and that $\mathscr{A}$ is closed under complementation and countable union.

If $E=\bigcap_{I} K_{i} \in \mathscr{K}^{\prime}$, each $K_{i} \in \mathscr{K}$, then $P(E)=\inf _{i \in I} P\left(K_{i}\right)$. Fix $\varepsilon>0$, and let $i \in I$ with $P\left(K_{i}\right)<P(E)+\varepsilon / 2$. Let $K \in \mathscr{K}$ with $K \subseteq K_{i}^{c}$ and $P(K)>$ $P\left(K_{i}^{c}\right)-\varepsilon / 2$. Put $u=K^{c}$. Then $E \subseteq E \subseteq u$ and $P(u \backslash E)<\varepsilon / 2+\varepsilon / 2=\varepsilon$, so $E \in \mathscr{A}$.

If $A \in \mathscr{A}$, and $\varepsilon>0$, let $E \subseteq A \subseteq u$ with $E \in \mathscr{K}^{\prime}, u \in \tau$, and $P(u \backslash E)<$ $\varepsilon$; then $u^{c} \subseteq A^{c} \subseteq E^{c}, u^{c} \in \mathscr{K}^{\prime}, E^{c} \in \tau$, and $P\left(u^{c} \backslash E^{c}\right)=P(u \backslash E)<\varepsilon$, so $A^{c} \in \mathscr{A}$.

If $A_{n} \in \mathscr{A}$ for $n \in \mathbb{N}$, and $\varepsilon>0$, let $E_{n} \subseteq A_{n} \subseteq u_{n}$ with $E_{n} \in \mathscr{K}^{\prime}$, $u_{n} \in \tau$, and $P\left(u_{n} \backslash E_{n}\right)<\varepsilon / 2^{n+1}$. Let $N \in \mathbb{N}$ with $P(E)>P\left(\bigcup_{n} E_{n}\right)-\varepsilon / 2$, where $E=\bigcup_{n \leq N} E_{n}$. Put $u=\bigcup_{n} u_{n}$. Then $E \subseteq \bigcup_{n} A_{n} \subseteq u$ and $P(u \backslash E) \leq$ $P\left(u \backslash \bigcup_{n} E_{n}\right)+\bar{P}\left(\bigcup_{n} E_{n} \backslash E\right)<\sum_{n} P\left(u_{n} \backslash E_{n}\right)+\varepsilon / 2<\varepsilon / 2+\varepsilon / 2=\varepsilon$, so $\bigcup_{n} A_{n} \in$ $\mathscr{A}$.

This proves that $P^{\prime}$ is $\mathscr{K}^{\prime}$-inner regular, and completes the theorem.

Remark. Suppose that $(S, \mathscr{A}, m)$ is an internal, finitely-additive ${ }^{*}$ measure space with $S \subseteq{ }^{*} X$ and $m\left(S \cap{ }^{*} K\right) \approx P\left({ }^{*} K\right)$ for every $K \in \mathscr{K}$. It is not difficult to see that the proof of Theorem 1.1(1) holds when $(S, L(\mathscr{A}), L(m))$ is everywhere substituted for $\left({ }^{*} X, L\left({ }^{*} \mathscr{B}\right), L\left({ }^{*} P\right)\right)$, using the restriction of $\varphi$ to $S$. This is a common setting in the applications of the Loeb measure.

\section{OPEN PROBLEMS}

Problem 1. Is Loeb measure compact?

This question, first posed in [10], remains open. Clearly Loeb measure is $\kappa$-compact if the nonstandard model is $\kappa^{+}$-saturated.

An affirmative answer would simplify the proof (in [10]) that Loeb measurable functions into metric spaces have liftings. It would also make it possible (using Theorem 1.1) to obtain every Loeb probability measure as the measurable image of a more saturated Loeb measure, a result that would prove useful.

The answer may well depend on the particular nonstandard model, or even on the existence of large cardinals.

Problem 2. Is the image of every Loeb measure compact?

More precisely, suppose $\varphi$ is a measure-preserving transformation from a Loeb probability space $(\Omega, L(\mathscr{A}), L(\mu))$ to a standard probability space $(X, \mathscr{B}, P)$; need $(X, \mathscr{B}, P)$ be a compact measure? An affirmative answer is, of course, the converse to Theorem 1.1.

Note that the answer is unknown even when $(X, \mathscr{B}, P)$ is a Radon measure on a Hausdorff topological space; however, if $X$ is regular and $\varphi$ has a lifting, then an affirmative answer follows from known results, e.g. in [4].

\section{REFERENCES}

1. S. Albeverio, J. E. Fenstad, R. Høegh-Krohn, and T. Lindstrøm, Nonstandard methods in stochastic analysis and mathematical physics, Academic Press, Orlando, 1986.

2. R. Anderson, Star-finite representations of measure spaces, Trans. Amer. Math. Soc. 271 (1981), 667-687. 
3. N. J. Cutland, Nonstandard measure theory and its applications, Bull. London Math. Soc. 15 (1983), 529-589.

4. D. Landers and L. Rogge, Universal Loeb-measurability of sets and of the standard part map with applications, Trans. Amer. Math. Soc. 304 (1987), 229-243.

5. T. Lindstrøm, Pushing down Loeb measures, 1981, Preprint Series, Matematisk Institutt, Univ. i Oslo, Mathematics No. 2, March 10.

6. P. A. Loeb, An introduction to nonstandard analysis and hyperfinite probability theory, Probabalistic Analysis and Related Topics II (A. T. Bharucha-Reid, ed.), Academic Press, New York, 1979.

7. T. Norberg, private correspondence.

8. __ Existence theorems for measures on continuous posets, with applications to random set theory, Report 1987-11, Dept. of Math., Univ. of Göteborg, 1987.

9. M. M. Rao, Projective limits of probability spaces, J. Multivariate Anal. 1 (1971), 28-57.

10. D. Ross, Measurable transformations in saturated models of analysis, $\mathrm{Ph} . \mathrm{D}$. Thesis, Univ. of Wisconsin, 1983.

11. W. Vervaat, Random upper semicontinuous functions and extremal processes, report MSR8801, Centrum voor Wiskunde en Informatica, 1988.

Department of Statistics, Oregon State University, Corvallis, Oregon 97331

Current address: Department of Mathematics, University of Hawaii at Manoa, Honolulu, Hawaii 96822 